\section{Acquired hemophilia A developing cerebral infarction 36 days after the frequent administration of bypass hemostatic agents}

\author{
Makoto Saito, ${ }^{1}$ Hajime Senjo, ${ }^{1}$ \\ Minoru Kanaya, ${ }^{1}$ Koh Izumiyama, ${ }^{1}$ \\ Akio Mori, ${ }^{1}$ Masanori Tanaka, ${ }^{1}$ \\ Masanobu Morioka, ${ }^{1}$ Masahiro Ieko ${ }^{2}$ \\ ${ }^{1}$ Department of Internal Medicine and \\ Hematology, Aiiku Hospital; \\ ${ }^{2}$ Department of Internal Medicine, \\ Health Sciences University of Hokkaido, \\ Japan
}

\begin{abstract}
A 74-years-old male who was a smoker and received treatment for hypertension, dyslipidemia, peripheral arterial disease and idiopathic interstitial pneumonia complained of subcutaneous hemorrhage of the right lower thigh. Marked anemia (hemoglobin $5.5 \mathrm{~g} / \mathrm{dL}$ ) and prolonged activated partial thromboplastin time ( $\geq 130$ seconds) were noted. The factor VIII activity level was reduced to $1.2 \%$, and the factor VIII inhibitor titer was $285.3 \mathrm{BU} / \mathrm{mL}$, a diagnosis of acquired hemophilia A (AHA) was made. Then, hematomas of 5 intra-muscles were recurred. Hemostasis became difficult despite frequent and high-dose administration of recombinant human coagulation factor VIIa (total: 18 days, $305 \mathrm{mg}$ ). Hemostasis was achieved by switching to activated prothrombin complex concentrate (for 3 days, 18,000 units), however, cerebral infarction occurred after 36 days. After the frequent administration of bypass hemostatic agents on elderly AHA patients with several risk factors for ischemic stroke, the risk of subsequent thrombotic events may persist for 1 month.
\end{abstract}

\section{Introduction}

Acquired hemophilia A (AHA) is an autoimmune hemorrhagic disease, whose pathogenesis involves a reduction in coagulation factor VIII (FVIII) activity related with the appearance of autoantibodies (inhibitors) against FVIII. ${ }^{1}$ AHA is rare, with an annual incidence of 1 per 1-4 million people. Elderly patients (age: $\geq 60$ years) account for $\geq 80 \%$ of patients. ${ }^{2}$ Furthermore, the underlying diseases include malignant tumors and autoimmune diseases, but idiopathic diseases account for approximately $50 \%$. $^{2,3}$

The treatment of AHA is divided into two points: immunosuppressive therapy to remove FVIII inhibitors and hemostatic therapy for hemorrhage. ${ }^{1}$ For hemostasis, bypassing agents, i.e. recombinant human coagulation factor VIIa (rFVIIa) and activated prothrombin complex concentrate (aPCC), are selected as first-line drugs. No comparative study on which preparation is more effective has been conducted, but the hemostatic effects of the two agents are favorable (response rate: $\geq 80 \%) .{ }^{4}$ However, serious thrombosis occurs as a complication once in a while, and we previously reported on the appropriate use of $\mathrm{rFVIIa}^{5}$ We report an instructive case with AHA in whom hemostasis was successfully achieved by frequent administration of rFVIIa and aPCC for repeated intramuscular hematomas, but cerebral infarction occurred after 36 days.

\section{Case Report}

\section{Patient presentation}

A 74-years-old Japanese male consulted a family doctor and hospitalized with subcutaneous hemorrhage with severe pain in the right lower thigh persisting for 2 days. Marked anemia [hemoglobin ( $\mathrm{Hb})$ : 5.5 $\mathrm{g} / \mathrm{dL}]$ and the prolongation of the activated partial thromboplastin time (APTT) $(\geq 130$ seconds) were noted. Red blood cell (RBC) and fresh frozen plasma were transfused. The patient was a smoker (a pack of cigarettes/day x 55 years) and had continuously undergone treatment for hypertension and dyslipidemia since 40 years of age. He had treated with cilostazol and nintedanib ethanesulfonate for peripheral arterial disease of the bilateral lower limbs and idiopathic interstitial pneumonia, respectively, for the past 6 months. He was referred to our hospital. On admission, anemia (Hb: 8.8 $\mathrm{g} / \mathrm{dL}$ ) and APTT prolongation (96.7 seconds) were observed, but the prothrombin time was normal (11.8 seconds). The APTT cross-mixing test showed a convex curve (upward) after incubation at $37^{\circ} \mathrm{C}$ for 2 hours. The patient was negative for lupus anticoagulant. The FVIII activity level was reduced to $1.2 \%$ and the FVIII inhibitor titer was 285.3 Bethesda units (BU)/mL. A diagnosis of AHA was made.

\section{Clinical course}

The clinical course is shown in Figure 1. Prednisolone (PSL; $1 \mathrm{mg} / \mathrm{kg}$ ) was administered as immunosuppressive therapy, and then, a large intramuscular hematoma occurred in the left mesogluteus muscle and
Correspondence: Makoto Saito, Department of Internal Medicine and Hematology, Aiiku Hospital, Minami 4 Nishi 25 Chuo-ku, Sapporo 064-0804, Japan.

Tel.: +81.11.563.2211 - Fax: +81.11.522.1691 E-mail: ikyoku@aiiku-hp.or.jp

Key words: Acquired hemophilia A; bypass hemostatic agents; cerebral infarction.

Conflicts of interests: the authors declare no conflict of interest.

Received for publication: 16 October 2017. Accepted for publication: 24 June 2018

This work is licensed under a Creative Commons Attribution-NonCommercial 4.0 International License (CC BY-NC 4.0).

(C) Copyright M. Saito et al., 2018

Licensee PAGEPress, Italy

Hematology Reports 2018; 10:7453

doi:10.4081/hr.2018.7453

anemia progressed (Hb: $4.3 \mathrm{~g} / \mathrm{dL}$ ). To achieve hemostasis, rFVIIa was administered, and there was no hemorrhage. The dose of rFVIIa was $90 \mu \mathrm{g} / \mathrm{kg}$ from the start of administration until the final dosing. In this month, it was administered 9 times for 3 days. After 1 month, intramuscular hematomas were repeatedly observed as described below, and anemia ( $\mathrm{Hb}: \geq 6 \mathrm{~g} / \mathrm{dL})$ protracted and RBC was transfused (a total dosage of 16 units). rFVIIa was frequently administered; i) recrudescence of the left mesogluteus muscle (Figure 2A) (rFVIIa was administered 13 times for 4 days), ii) the right trapezius muscle (Figure 2B) and iii) latissimus dorsi muscle (Figure 2C) (17 times for 5 days). At this point, RBC transfusion was not required. However, pain related with repeated intramuscular hematomas was severe, and the patient wished to undergo prompt hemostasis. Additionally, rFVIIa was administered to treat intramuscular hematomas; in iv) the left thoracic wall (not shown) (6 times for 2 days), v) the right iliac muscle (Figure 2D) and recrudescence of the above (4) left thoracic wall (16 times for 4 days). However, after 3 days, additional hemorrhage from (1) the left mesogluteus muscle was observed, and hemostatic control with rFVIIa was considered to be difficult (total dose: $305 \mathrm{mg}$ ). After obtaining informed consent from the patient, aPCC at 60 units $(\mathrm{U}) / \mathrm{kg}$ was administered 6 times (for 3 days, total dose: 18,000 U). There was no hemorrhage afterwards, and the $\mathrm{Hb}$ level increased from 8.1 to $12.1 \mathrm{~g} / \mathrm{dL}$ after 2 weeks.

During this period, PSL decreased the 
FVIII inhibitor titer slowly, and rituximab (RTX, $375 \mathrm{mg} / \mathrm{m}^{2}$ ) was combined 6 times from 1 month after the start of PSL therapy. High-dose cyclophosphamide (CPA, 1 g) was administered once 1 month after the start of combination therapy with RTX. As a result, FVIII inhibitors were completely removed 15 weeks after the start of PSL therapy (FVIII activity level: $64.4 \%$ ). At this time, 36 days after the completion of aPCC administration, motor paralysis of the left lower limb was exhibited. Magnetic resonance imaging (MRI) of the brain revealed the onset of cerebral infarction involving the right parietal lobe (Figure 3A and $\mathrm{B}$ ). Later, detailed examination at the designated neurosurgery clinic demonstrated marked arteriosclerosis-related stenosis of the right internal carotid and anterior cerebral arteries, suggesting atherothrombotic brain infarction. The administration of lowdose cilostazol (100 mg/day) which was discontinued since his admission was resumed, and the infarcted focus disappeared after 2 months. Rehabilitation for 3 months reduced the sequelae of the cerebral infarction, and he was discharged. After 3 weeks, PSL was discontinued. During the 15-months follow-up after discharge, there has been no recrudescence of AHA or cerebral infarction. The patient is being followed-up at the outpatient with a performance status of 1 .

\section{Discussion}

Amano et al. reported that both the median (mean) frequency of rFVIIa administration [5.0 (14.8) times] and treatment period (2.0 (3.4) days) in patients with intramuscular hemorrhage were significantly higher (longer) than in other hemorrhagic sites. ${ }^{6}$ According to the EACH2 Registry study, the median total frequency of administration was 12 (3-35) times in 174 patients treated with rFVIIa, and the median total dose of rFVIIa was 84 (24-216) $\mathrm{mg} .{ }^{4}$ In the case presented here, frequent and high-dose rFVIIa was administered 61 times (total dosage: $305 \mathrm{mg}$ ) for a total of 18 days to treat repeated intramuscular hematomas, but hemostasis was not achieved. Then, rFVIIa was switched to aPCC, leading to hemostasis. It is difficult to predict which of the two drugs, rFVIIa or aPCC, is more effective for hemostasis. Furthermore, a consensus regarding the timing of switching has not been reached. In our case, if rFVIIa had been switched to aPCC earlier, hemostasis may have been achieved earlier, reducing the dose of rFVIIa.

As AHA frequently develops in elderly

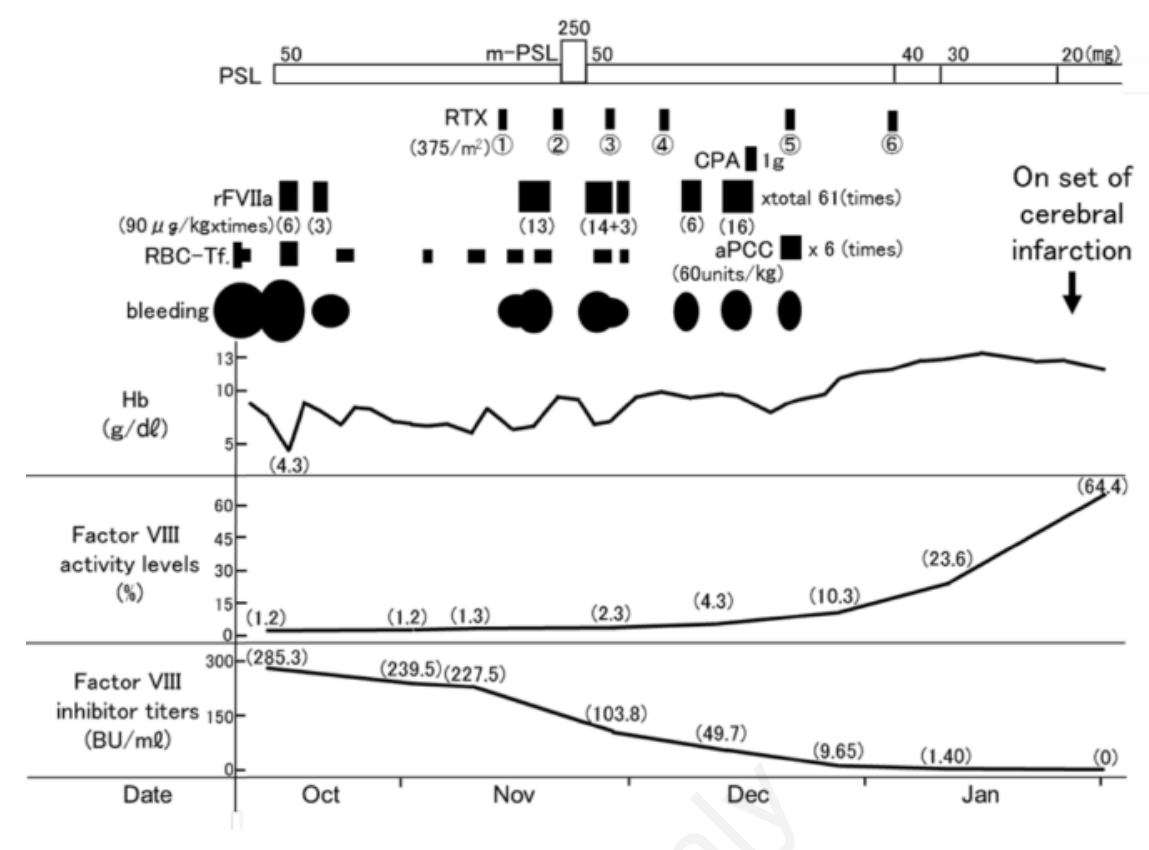

Figure 1. Clinical course of the patient. Hb, hemoglobin; RBC-Tf., red blood cell transfusion; rFVIIa, recombinant human coagulation factor VIIa; aPCC, activated prothrombin complex concentrate; PSL, prednisolone; m-PSL, methylprednisolone; RTX, rituximab; CPA, cyclophosphamide.
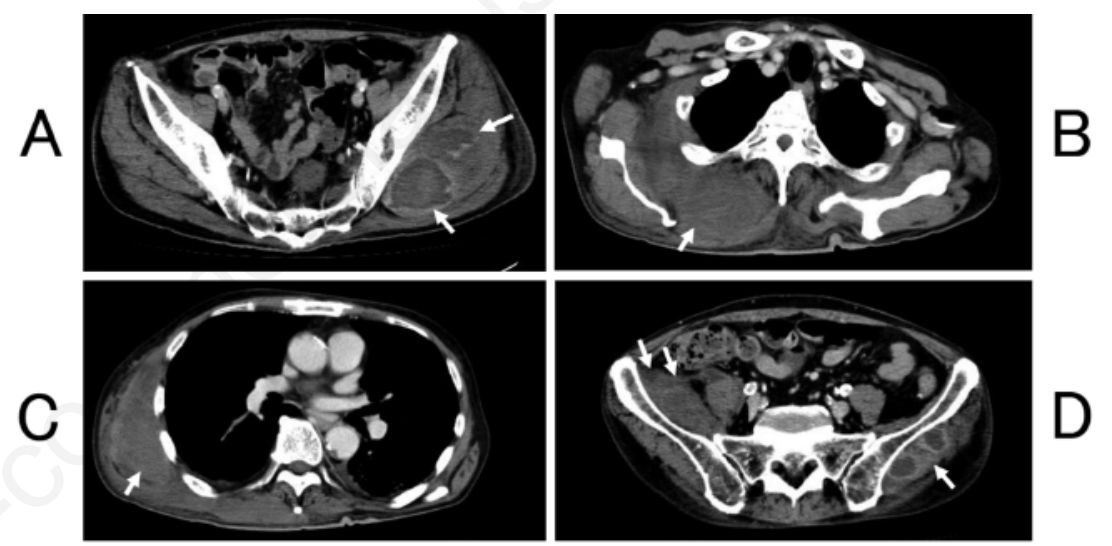

Figure 2. Intramuscular hemorrhage on CT (white arrow). A) Left middle gluteal muscle; B) right trapezius muscle; C) right latissimus dorsi muscle; D) right iliac muscle (intramuscular hemorrhage of the left middle gluteal muscle was also observed).

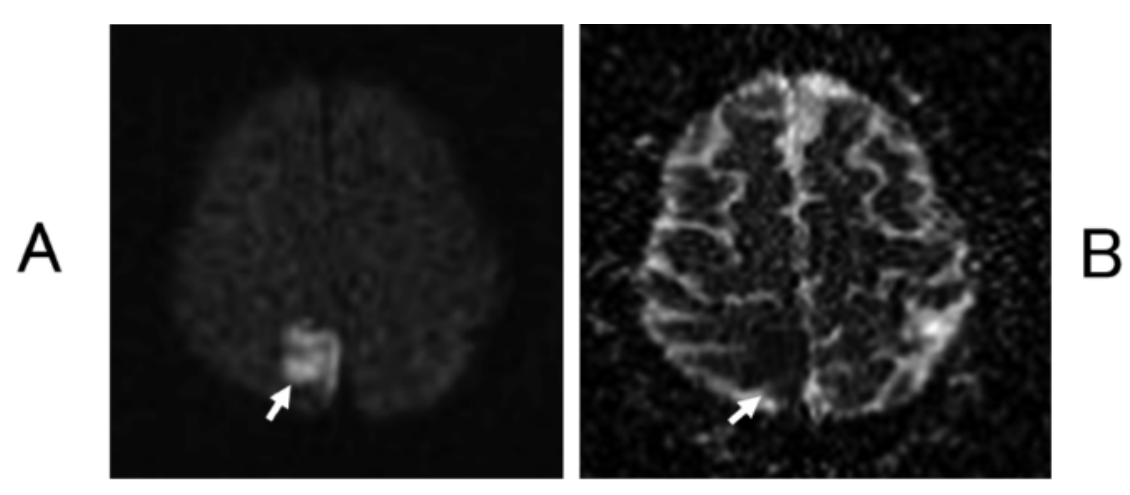

Figure 3. Brain MRI. A) A diffusion-weighted image showed a high-signal-intensity lesion in the right parietal lobe. B) An apparent diffusion coefficient (ADC) map indicated a low-signal-intensity lesion, suggesting the new onset of cerebral infarction. 
patients, arteriosclerotic diseases, such as hypertension, diabetes mellitus, and dyslipidemia, are thought to be present in several cases. ${ }^{5}$ Recent studies indicated thrombotic complications in 2.9 to $6.5 \%$ of AHA patients treated with rFVIIa may be occurred, especially after long-term highdose rFVIIa administration or after the start of combination therapy with aPCC without a sufficient interval. ${ }^{5-7}$ According to Abshire's study, the interval from the start of rFVIIa administration until the onset of thrombotic events was 2 hours to 5 days in patients with acute myocardial infarction, 9 hours to 22 days in those with cerebrovascular accidents/thrombosis, and 2 days to 1 month in those with deep vein thrombosis/pulmonary embolism. ${ }^{8}$ In the EACH2 Registry study, hemostatic-treatment-associated thrombotic events occurred in 11 of 307 patients $(3.6 \%) .{ }^{4}$ Of these, stroke was noted in only 1 , myocardial infarction in 6 , and venous thromboembolism in 4 . The incidence of thromboembolism, especially cerebral infarction, related with $\mathrm{rFVIIa/aPCC}$ administration at a standard dose may be very rare.

Resembling our patient, a previous study reported a 69-years-old male with AHA who developed ischemic stroke after rFVIIa administration. ${ }^{9}$ For this patient, rFVIIa was administered at 3-hour intervals for 3 days, increasing the FVIII activity level to $97.56 \%$ at the end of the first week (pretreatment value: $1.68 \%$ ); hemostasis was successfully achieved. However, acute cerebral infarction occurred 6 days after the completion of rFVIIa treatment. Thus, in AHA patients, increased plasma FVIII activity after inhibitor eradication was found to be an independent risk factor for thrombosis. ${ }^{10}$ Our patient was the age of 74years-old male and had a habit of smoking over $\geq 50$ years. He had received treatment for hypertension and dyslipidemia over $\geq 30$ years, and had a peripheral arterial disease of the lower limbs. He was suggesting the progression of systemic arteriosclerosis, indeed, marked stenosis of the right internal carotid and anterior cerebral arteries was observed. Pre-atherothrombotic cerebral infarction state may have been present, regardless of AHA onset. The function of the platelet may be enhanced against marked hemorrhage. In addition, the frequent and high-dose administration of bypass hemostatic agents may have promoted platelet thrombus formation, and may have led to ischemic stroke. Furthermore, the direct involvement of bypass hemostatic agents, as well as the rapid increase in FVIII activity related with FVIII inhibitor eradication, may have resulted in hyper-coagulation. Clinicians must be aware that the bleeding tendency in AHA patients may quickly shift to thrombus formation during the rapid recovery of FVIII activity.

\section{Conclusions}

In conclusion, if hemostasis is difficult despite frequent administration of rFVIIa for AHA patients with repeated intramuscular hematomas, hemostasis may be achieved earlier by switching rFVIIa to aPCC. When hemostasis is achieved with the frequent and high-dose administration of bypass hemostatic agents in elderly AHA patients with several risk factors for ischemic stroke, the risk of subsequent thrombotic events may persist for 1 month. In particular, thrombus formation may occur during the rapid recovery of FVIII activity.

\section{References}

1. Delgado J, Jimenez-Yuste V, Hernandez-Navarro F, Villar A. Acquired haemophilia: review and meta-analysis focused on therapy and prognostic factors. $\mathrm{Br} \mathrm{J}$ Haematol 2003; 121: 21-35.

2. Collins PW, Hirsch S, Baglin TP, et al. Acquired hemophilia $\mathrm{A}$ in the United
Kingdom: a 2-year national surveillance study by the United Kingdom Haemophilia Centre Doctors' Organisation. Blood 2007;109:1870-7.

3. Morrison AE, Ludlam CA, Kessler C. Use of porcine factor VIII in the treatment of patients with acquired hemophilia. Blood 1993;81:1513-20.

4. Baudo F, Collins P, Huth-Kühne A, et al. Management of bleeding in acquired hemophilia A: results from the European Acquired Haemophilia (EACH2) Registry. Blood 2012;120:3946.

5. Saito M, Kanaya M, Izumiyama K, et al. Treatment of bleeding in acquired hemophilia A with the proper administration of recombinant activated factor VII: single-center study of 7 cases. Int J Gen Med 2016;9:393-9.

6. Amano K, Seita I, Higasa S, et al. Treatment of acute bleeding in acquired haemophilia A with recombinant activated factor VII: analysis of 10-year Japanese postmarketing surveillance data. Haemophilia 2017;23:50-8.

7. Di Capua M, Coppola A, Nardo A, et al. Management of bleeding in acquired haemophilia A with recombinant activated factor VII: does one size fit all? A report of four cases. Blood Transfus 2015;13:328-32.

8. Abshire T, Kenet G. Recombinant factor VIIa: review of efficacy, dosing regimens and safety in patients with congenital and acquired factor VIII or IX inhibitors. J Thromb Haemost 2004;2:899-909.

9. Katgi A, Ataca P, Kahraman S, et al. Ischemic stroke after recombinant factor VIIa treatment in acquired hemophilia A patient. Blood Coagul Fibrinolysis 2012;23:69-70.

10. Nicolaides AN, Breddin HK, Carpenter $\mathrm{P}$, et al. Thrombophilia and venous thromboembolism. International consensus statement. Guidelines according to scientific evidence. Int Angiol 2005;24:1-26. 